\title{
Modelling the elastic properties of the anterior eye and their contribution to maintenance of image quality: the role of the limbus
}

\begin{abstract}
Purpose The elastic moduli of the cornea, sclera, and limbus for different corneal eccentricities $(e)$ and varying levels of intraocular pressure (IOP) were modelled in order to determine how the rheological properties, especially those of the limbus, need to alter to maintain optical image quality when the eye is subjected to small variations in IOP.

Methods Finite element analysis (FEA) was used to construct eyeball models with four different corneal eccentricities $(e=0,0.33,0.5$, 0.65). Three values for Young's modulus of the cornea were tested in all models $(0.2$ megapascal (MPa), 1.2 and 10 MPa). For each corneal modulus, scleral moduli of $3,4,5,7$, and 10 times that of the corneal modulus were selected. The limbal modulus was varied to optimise image quality of the eye model subjected to IOP variations of $\pm 0.8 \mathrm{mmHg}$ for three different levels of IOP $(8,16$, and $32 \mathrm{mmHg}$ ).

Results The elastic modulus of the limbal ring increases with an increase in corneal modulus and rises to a peak when the ratio of scleral to corneal moduli is between 5 and 7 depending on corneal eccentricity. Different levels of IOP produce only slight differences in the relative moduli required to maintain optical image quality.

Conclusions The significance of a peak in the value of Young's modulus of the limbus is not clear but suggests that there may be an optimal limbal modulus that must be balanced with the moduli of cornea and sclera for preservation of image quality.
\end{abstract}

Eye (2007) 21, 1087-1094; doi:10.1038/sj.eye.6702464; published online 23 June 2006

M Asejczyk-Widlicka' ${ }^{1}$ DW Śródka², H Kasprzak ${ }^{3}$ and BK Pierscionek ${ }^{3}$

Keywords: cornea; sclera; limbus; intraocular pressure; biomechanics; finite element analysis

\section{Introduction}

The quality of the image that falls on the retina depends on the integrity of the optics of the eye: transparency of the media and refractive characteristics of the lens and cornea. Optical integrity depends in part on rheological factors: how the ocular tissues (cornea, sclera, and lens) deform in response to force applied. The ratio of force, or stress, to deformation, or strain, is termed the elastic (Young's) modulus. The importance of these properties is becoming more evident as findings indicate that the outer coat of the eye is more malleable than had previously been thought. Studies have found that accommodation of the lens can induce slight but measurable changes in the central corneal radius of curvature. ${ }^{1}$ Subsequent studies have supported this ${ }^{2}$ while others have shown that accommodation can produce changes in the shape and size of the globe. ${ }^{3,4}$ Perhaps the most evident manifestation of ocular pliability throughout life is the development of myopia that is increasingly found to occur in adults. ${ }^{5-8}$ This is most likely to be attributable to accommodative effort but may also involve a second growth spurt in adult life. ${ }^{5}$

Accommodation is not the only factor that may influence the shape and size of the globe in adult life. A change in intraocular pressure (IOP), if significant enough, has the potential to alter eyeball length and corneal shape.

Elevations in the IOP can produce characteristic signs and symptoms that help to provide a
${ }^{1}$ School of Biomedical Sciences, University of Ulster, Coleraine, Northern Ireland, UK

${ }^{2}$ Faculty Division of Deformable Bodies Mechanics, Wroclaw University of Technology, Wroclaw, Poland

${ }^{3}$ Institute of Physics, Wroclaw University of Technology, Wroclaw, Poland

Correspondence: BK Pierscionek, School of Biomedical Sciences, University of Ulster, Cromore Road, Coleraine, Northern Ireland BT52 1SA, UK

Tel: 02870323293;

Fax: 02870324965

E-mail: b.pierscionek@ ulster.ac.uk

Received: 24 October 2005 Accepted in revised form: 27 April 2006 Published online: 23 June 2006 
diagnostic picture of glaucoma. However, IOP can also increase with no obvious ocular or visual disturbances. Any pressure-induced changes to the eyeball and/or its components should alter the optics of the eye, yet the quality of the retinal image appears, in some cases of raised IOP, to be undisturbed. This suggests that there may be a compensating mechanism that adjusts the optics of the eye to prevent changes in the IOP from disturbing image quality.

Any adjustment of ocular optics in response to a change in IOP requires an appropriate balance of elastic moduli of the cornea, sclera, and limbus. An elastic modulus of the cornea that is relatively low and easily deformable would lead to rapid and excessive shape changes with consequent detrimental effects on visual imagery. Conversely, a high corneal stress to strain ratio would render the eyeball rigid and unable to adjust to any pressure whether internal (IOP) or external (such as the shape change on which successful orthokeratology depends). In the eyeball, which has sufficient elasticity for shape adjustment yet which is required to maintain optical quality, a structural feature that provides some restraint and helps to control corneal curvature may be expected. Such a role has been ascribed to the limbal region by Maurice ${ }^{9}$ who speculated that the limbus forms a rigid ring that supports the curvature of the cornea. Some support for this notion was provided by studies on bovine tissue $\mathrm{e}^{10}$ and there is anatomical evidence for the existence of such a ring based on the preferential orientation of collagen fibres in the limbal region. ${ }^{11,12}$ and on the distribution of epithelial stem cells. ${ }^{13}$ Hjortdal $^{14}$ disputed the notion of perfect rigidity but acknowledged that the limbus may function as a reinforcing structure in the circumferential direction. Asejczyk-Widlicka et $a l^{15}$ further proposed that the limbus may act to adjust for IOP-induced shape changes in the cornea in order to maintain image quality on the retina.

This paper presents the results of modelling the elastic moduli of the cornea, sclera, and limbus to determine whether there may be an optimum set of rheological values required for maintenance of ocular image quality when the eye is subjected to small variations in IOP.

\section{Method}

The eye model was created using COSMOS/M Finite Element Analysis (v. 1.75, Structural Research \& Analysis Corporation) with three defined rheological regions for the outer shell, representing the cornea, sclera, and limbal ring. The cornea and sclera were constructed from 288 elements of the 20-node solid 3D type and the limbal ring from eight rod type elements. Figure 1 shows a half-globe model. The posterior hemisphere of the sclera was fixed inside the orbit, to mimic the constraint on

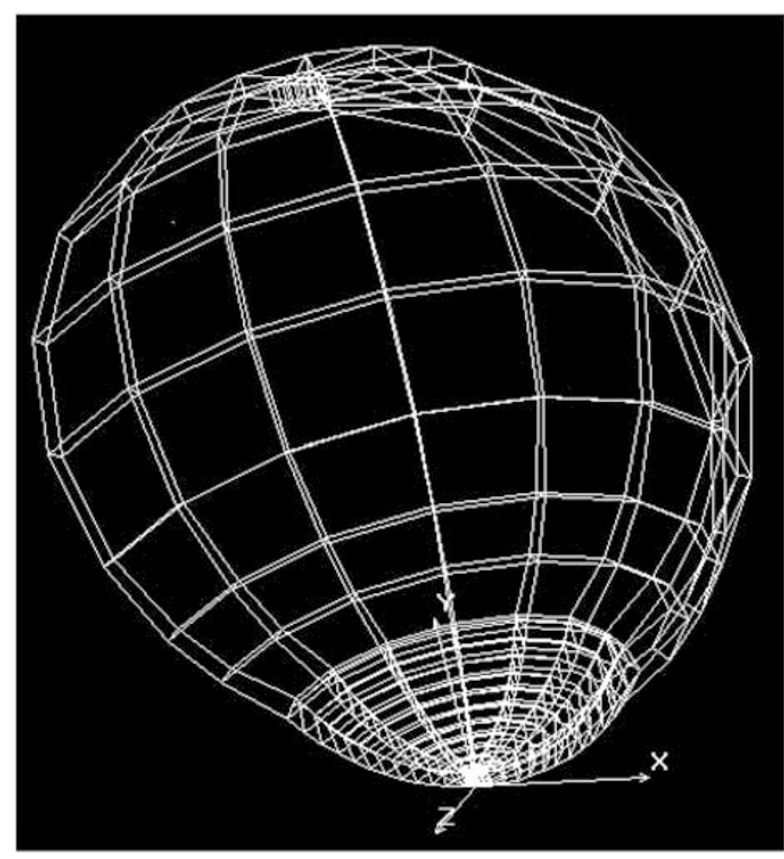

Figure 1 Finite element model of the globe of the eye, showing cornea, sclera, and limbus.

Table 1 The parameters of the eyeball model

Parameters used in the finite element model

\begin{tabular}{ll}
\hline Parameter & Value \\
\hline Axial radius of anterior corneal curvature & $R_{\mathrm{a}}=7.86 \mathrm{~mm}$ \\
Axial radius of posterior corneal curvature & $R_{\mathrm{p}}=6.76 \mathrm{~mm}$ \\
Central corneal thickness & $d_{c}=0.52 \mathrm{~mm}$ \\
$\begin{array}{l}\text { Peripheral corneal thickness adjacent to } \\
d_{\mathrm{p}}=0.65 \mathrm{~mm}\end{array}$ \\
$\quad$ Dimbus & $11.5 \mathrm{~mm}$ \\
Diameter of the cornea & $n_{r} \cong 1.3771$ \\
Average corneal refractive index & $n \cong 1.336$ \\
$\quad$ humour and vitreous body & $\begin{array}{l}\text { lens } \cong 22.07 \mathrm{D} \\
\text { Refractive power of lens }\end{array}$ \\
Poisson ratio & $v=0.45$ \\
\hline
\end{tabular}

Values are taken from the literature. ${ }^{16-18}$

elongation in the posterior direction. Nodes, which lie on the symmetry plane, have freedom of displacement only in this plane. The eye was maintained in the unaccommodated state. Parameters of the model are shown in Table $1 .^{16-18}$ Poisson's ratio is taken as $0.45^{15}$

The anterior and posterior corneal curvatures are approximated by a conic section: ${ }^{16}$

$$
z(x)=\frac{1}{e^{2}-1}\left[\sqrt{R_{0}^{2}+x^{2}\left(e^{2}-1\right)}-R_{0}\right]
$$


where $e$ denotes the eccentricity and $R_{0}$ is the mean axial radius of curvature $\left(R_{0}=7.86 \mathrm{~mm}\right.$ for the anterior surface and $6.76 \mathrm{~mm}$ for the posterior surface).

The refractive power of the eye $\left(P_{\text {eye }}\right)$ is given by:

$$
P_{\text {eye }}=P_{\text {cornea }}+P_{\text {lens }}-\frac{d P_{\text {cornea }} P_{\text {lens }}}{n}
$$

where $P_{\text {cornea }}$ is the power of the cornea, $P_{\text {lens }}$ is the power of the lens, $d$ is the distance between the secondary principal plane of the cornea and the primary principal plane of the lens, $n$ is the refractive index of the ocular media (aqueous and vitreous $=1.336$ ) and the focal length of the eye is:

$$
f=\frac{n}{P_{\text {eye }}}
$$

The change in the axial length of the eyeball $\Delta l$ induced by slight variations in IOP $( \pm 0.8 \mathrm{mmHg})$ is defined as:

$$
\Delta l=a_{1}+\Delta S_{\mathrm{H}}^{\prime}
$$

where $a_{1}$ is the displacement of the limbus, $\Delta S_{\mathrm{H}}{ }^{\prime}$ is the change in distance between secondary principal plane of the eye $\left(H_{\mathrm{e}}{ }^{\prime}\right.$ in Figure 2 and that of the lens $\left(H_{1}^{\prime}\right.$ in Figure 2)

The quantity $a_{1}$ is obtained from finite element analysis (FEA) while $S_{\mathrm{H}}{ }^{\prime}$ is given by:

$$
S_{\mathrm{H}}^{\prime}=d \frac{P_{\text {cornea }}}{P_{\text {eye }}}
$$

A change in the position of the limbus in response to a variation in IOP will affect the retinal image. ${ }^{19}$ Defocus can therefore be described as a function of IOP:

$$
B(p)=\Delta l(p)-\Delta f(p)
$$

where $B(p)$ is the axial displacement of the focal point as a function of IOP $(p), p$ is the IOP normalised to a dimensionless variable acting as a multiplier of the nominal pressure $(8 \mathrm{mmHg}$ is denoted by $p=0.5$; $16 \mathrm{mmHg}$ is denoted by $p=1 ; 32 \mathrm{mmHg}$ by $p=2$ ). The

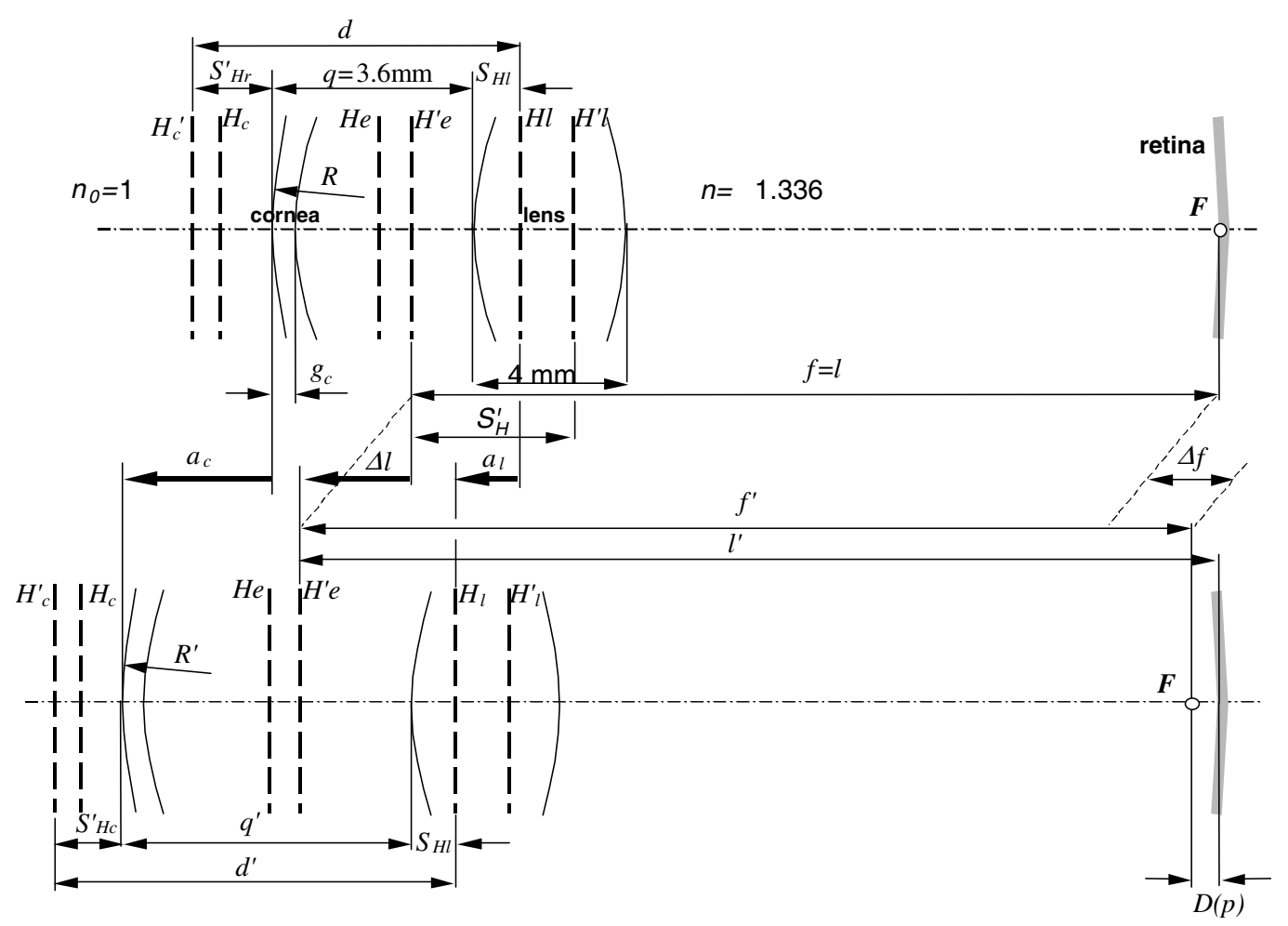

Figure 2 The reduced optical system of the eye model. $H_{\mathrm{e}}$ and $H_{e}^{\prime}$ are first and second principal planes of the eye; $\left(H_{\mathrm{c}}, H_{c}^{\prime}\right)$ and $\left(H_{1}, H_{1}^{\prime}\right)$ are first and second principal planes of cornea and lens, respectively; $n_{0}$ is the refractive index of ambient air; $n$ is refractive index of aqueous humour and vitreous body; $d$ is the distance between the second principal plane of the cornea and the first principal plane of the lens; $a_{\mathrm{c}}$ is the displacement of the corneal vertex affected by variations in IOP; $\Delta l$ is the displacement of the second principal plane of the eye $\left(H_{\mathrm{e}}{ }^{\prime}\right)$ after a change in IOP; $a_{1}$ is the displacement of the limbus; $S_{\mathrm{H}}{ }^{\prime}$ is the distance from the second principal plane of the eye to the second principal plane of the lens; $f, f$ are the focal lengths of the eye before and after IOP change, respectively; $\Delta f$ is the change in the focal length of the eye. 
variation in IOP applied to the models is $\Delta p= \pm 0.05$ $( \pm 0.8 \mathrm{mmHg})$ at each level of IOP.

For maintenance of optimal image quality the following criterion must be met:

$$
\frac{\mathrm{d} B}{\mathrm{~d} p}=0
$$

This is achieved by adjusting Young's modulus of the limbal ring to a value that supports the necessary change in corneal curvature required to compensate for the change in axial length of the eye. ${ }^{15}$ The procedure was applied to models with different corneal eccentricities, varying moduli of the cornea and sclera and for three levels of IOP.

\section{Results}

Three values of Young's modulus for the cornea: 0.2, 1.2, and $10 \mathrm{MPa}$, sampling the wide range of values reported in the literature, were selected. ${ }^{20-26}$ The scleral modulus was taken as greater than that of the cornea ${ }^{27}$ by $3,4,5,7$, and 10 times $\left(E_{\text {sclera }} / E_{\text {cornea }}=3,4,5,7\right.$, and 10$)$. Models were created for four different corneal eccentricities: $e=0$ (circle), $e=0.33, e=0.5$, and $e=0.65$ and calculations made for three levels of IOP representing 8,16 , and $32 \mathrm{mmHg}$.

The results are shown in Figures 3-6. In all figures, the limbal modulus is plotted against that of the sclera for each of three values of IOP. Figure 3 applies to a spherical cornea $(e=0)$ and in all cases the limbal modulus rises to a peak when the ratio $E_{\text {sclera }} / E_{\text {cornea }}$ is around five at which point it is about half the value of the scleral modulus. For corneal moduli of 0.2 and 1.2 (Figure 3a and $b$, respectively) there is little variation for the different values of IOP. For a corneal modulus of $10 \mathrm{MPa}$ (Figure 3c) the peak value is higher for IOP $=32 \mathrm{mmHg}$ than for lower levels of IOP.

For $e=0.33$ (Figure 4), the curves are similar in shape to those in Figure 3. Again there is a peak in limbal modulus value and this occurs when $E_{\text {sclera }} / E_{\text {cornea }}$ is around 5-6. At this point the limbal modulus is about two-thirds that of the scleral modulus and there is little variation for different values of IOP. For a corneal modulus of $0.2 \mathrm{MPa}$ (Figure 4a), the limbal modulus is slightly higher for IOP $=32 \mathrm{mmHg}$ than for the other two IOP values.

In Figure $5(e=0.5)$ the curves are less symmetrical than those in Figures 3 and 4. Again there is a peak value for the limbal modulus, which is reached when $E_{\text {sclera }} /$ $E_{\text {cornea }}$ is around 6-7, and at this point it is slightly higher than that of the sclera. There are only slight variations for different levels of IOP.
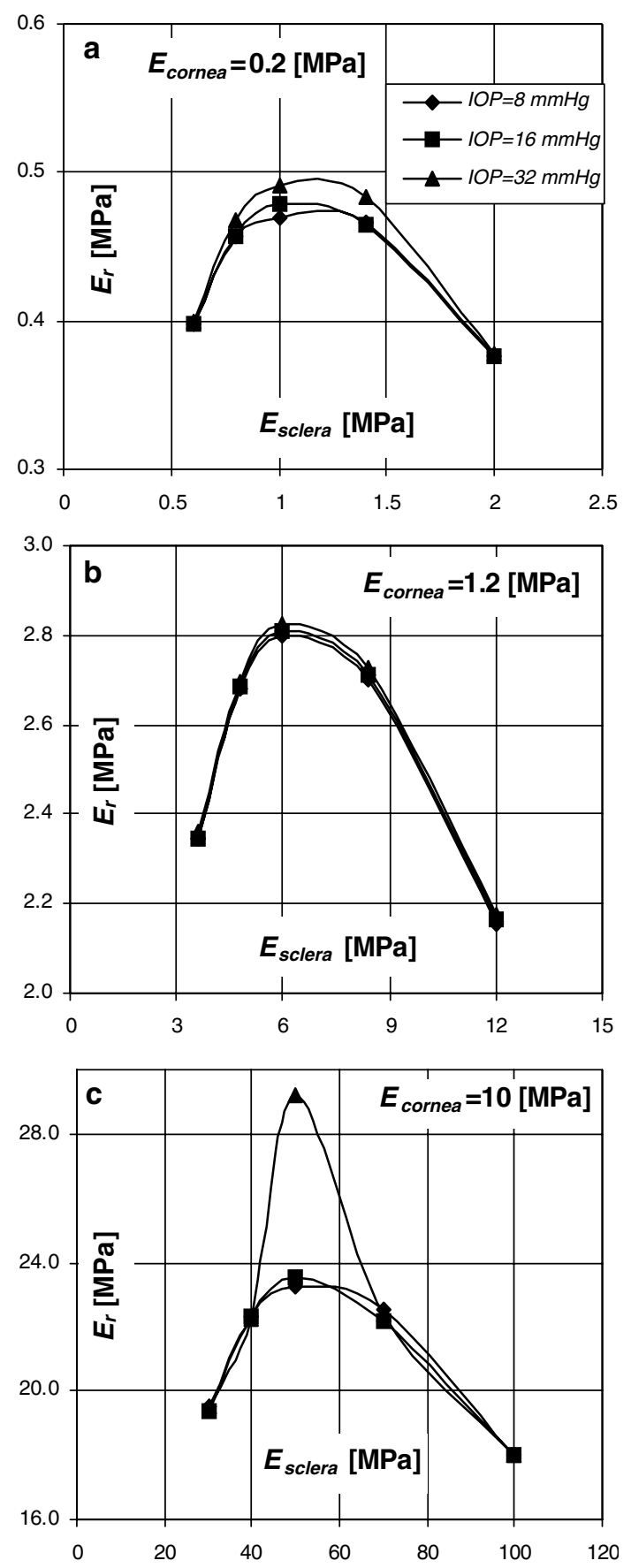

Figure 3 Young's modulus of the limbal ring $\left(E_{\mathrm{r}}\right)$ plotted as a function of scleral modulus $\left(E_{\mathrm{s}}\right)$ for corneal moduli $\left(E_{\mathrm{c}}\right)$ of (a) $0.2 \mathrm{MPa}$; (b) $1.2 \mathrm{MPa}$; and (c) $10 \mathrm{MPa}$ and for three levels of IOP $(8,16,32 \mathrm{mmHg})$. Corneal eccentricity $(e)=0$.

For $e=0.65$ (Figure 6), the peak limbal modulus is reached when $E_{\text {sclera }} / E_{\text {cornea }}$ is around 6-7 and at this point it is around 3-4 times higher than the scleral modulus. For a corneal modulus of $0.2 \mathrm{MPa}$ (Figure 6a), the limbal modulus is higher when IOP $=32 \mathrm{mmHg}$ compared to the other values of IOP. 

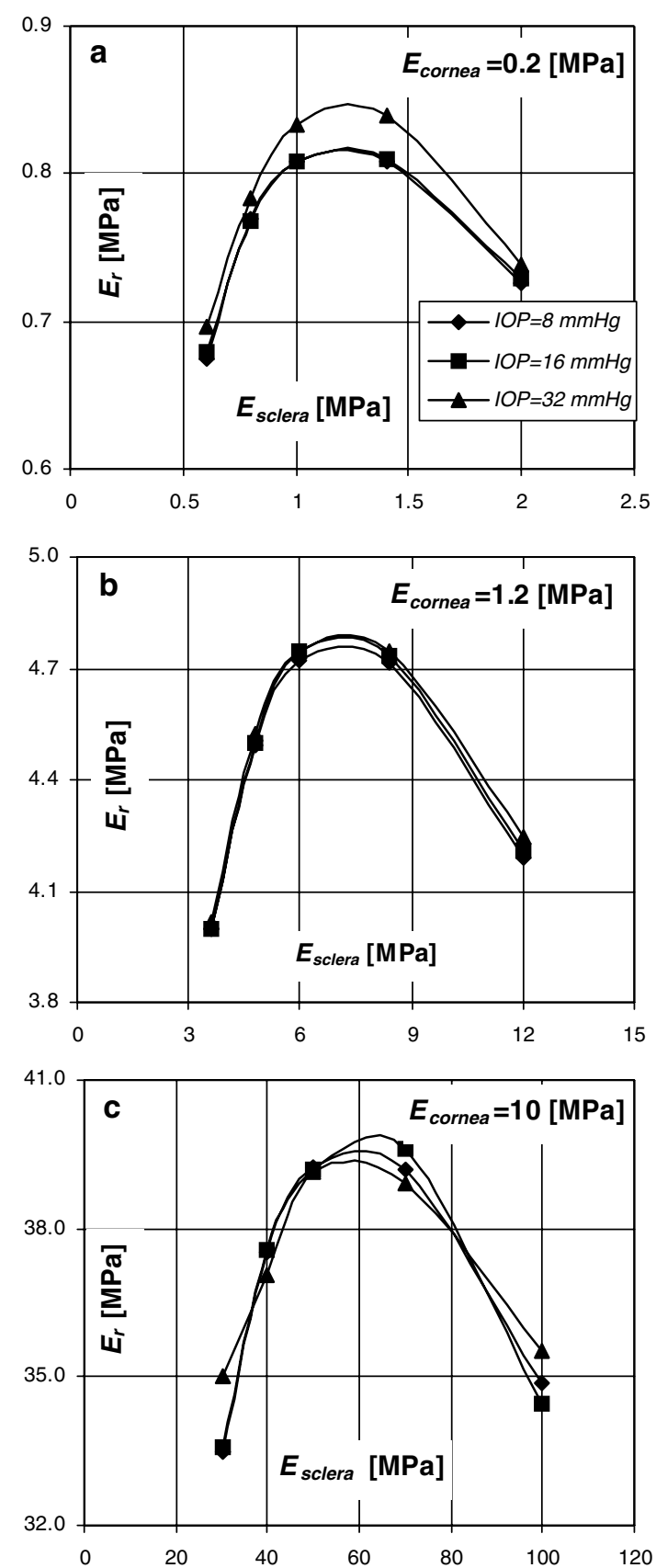

Figure 4 Young's modulus of the limbal ring $\left(E_{\mathrm{r}}\right)$ plotted as a function of scleral modulus $\left(E_{\mathrm{s}}\right)$ for corneal moduli $\left(E_{\mathrm{c}}\right)$ of (a) $0.2 \mathrm{MPa}$; (b) $1.2 \mathrm{MPa}$; and (c) $10 \mathrm{MPa}$ and for three levels of IOP $(8,16,32 \mathrm{mmHg})$. Corneal eccentricity $(e)=0.33$.

\section{Discussion}

The significance of ocular mechanics to optics of the eye has important implications for postsurgical changes to material properties that may alter the refractive power, as well as for understanding the dynamics of a healthy eye that needs to maintain ocular image quality. Liu and
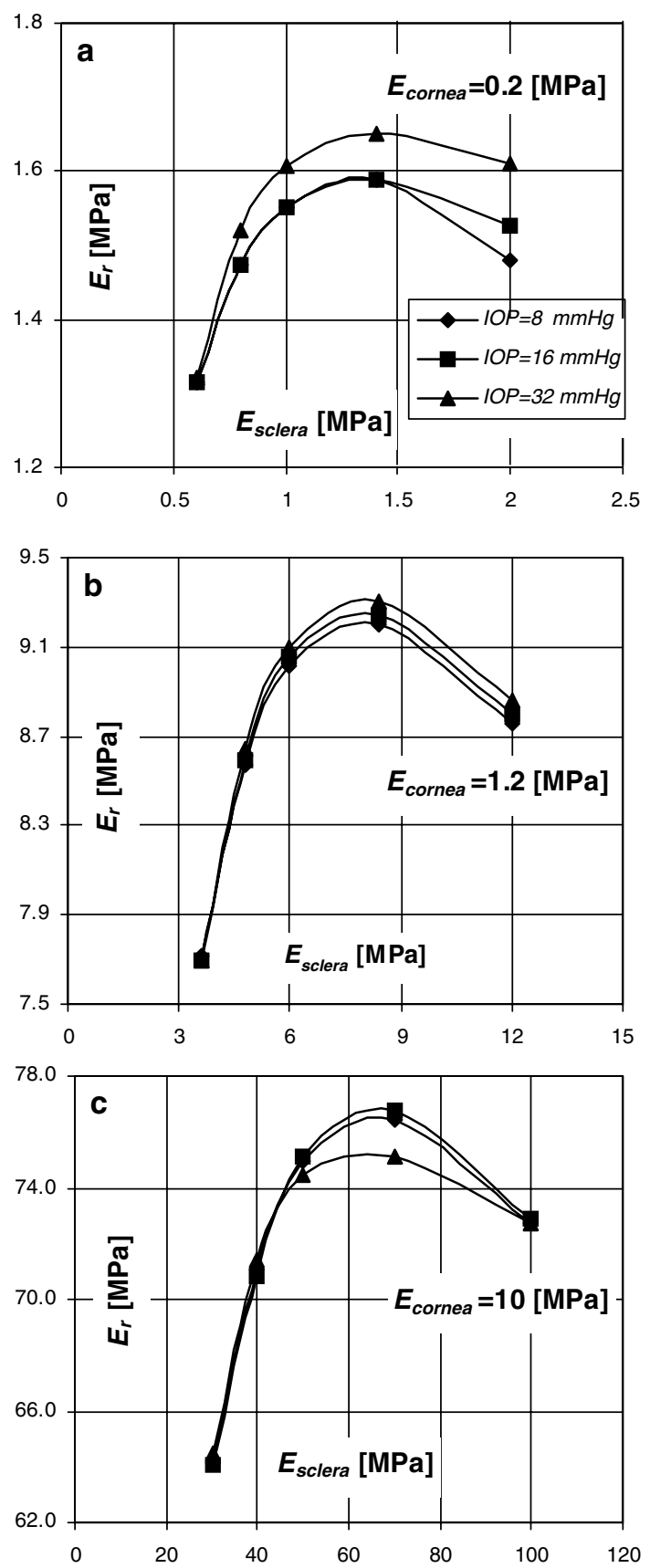

Figure 5 Young's modulus of the limbal ring $\left(E_{\mathrm{r}}\right)$ plotted as a function of scleral modulus $\left(E_{\mathrm{s}}\right)$ for corneal moduli $\left(E_{\mathrm{c}}\right)$ of $(\mathrm{a})$ $0.2 \mathrm{MPa}$; (b) $1.2 \mathrm{MPa}$; and (c) $10 \mathrm{MPa}$ and for three levels of IOP $(8,16,32 \mathrm{mmHg})$. Corneal eccentricity $(e)=0.5$.

Roberts ${ }^{28}$ have modelled the rheological properties of the cornea and found that these may have a greater influence on the measurement of IOP than the corneal shape or thickness. Obtaining accurate values for Young's moduli of the cornea, sclera, and limbal region is, however, frustrated by difficulties in methodology. 

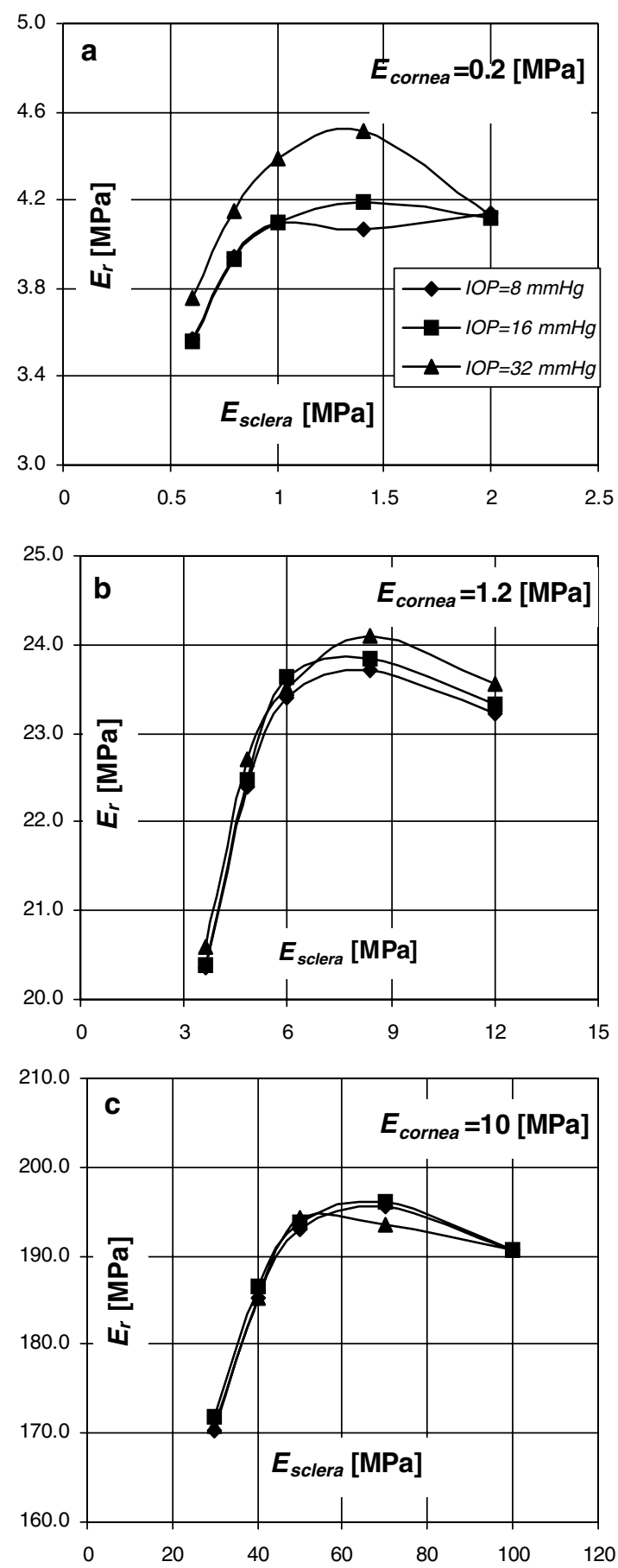

Figure 6 Young's modulus of the limbal ring $\left(E_{\mathrm{r}}\right)$ plotted as a function of scleral modulus $\left(E_{\mathrm{s}}\right)$ for corneal moduli $\left(E_{\mathrm{c}}\right)$ of (a) $0.2 \mathrm{MPa}$; (b) $1.2 \mathrm{MPa}$; and (c) $10 \mathrm{MPa}$ and for three levels of IOP $(8,16,32 \mathrm{mmHg})$. Corneal eccentricity $(e)=0.65$.

Measurements of material properties have produced a large number of inconsistencies with reported values of Young's modulus for the in vivo cornea varying from $0.026^{26}$ to $9.03 \mathrm{MPa}{ }^{22}$ In vitro results have shown even greater variations: Andreassen et $a l^{21}$ reported an elastic modulus value of $57 \mathrm{MPa}$ for corneal strips. This is not surprising given the wide range of experimental techniques and conditions, extent of post-mortem changes (for in vitro samples), as well as variations in tissue preparation and levels of hydration at the time of measurement. Indeed, some of the results may not be relevant to the physiological state of the tissue. In particular any procedure that involves dissection and/or freezing and thawing can significantly alter structural properties. Furthermore, tissue that is formed into a curved structure in vivo, as are the cornea and sclera, may undergo some alteration to its material properties when it is straightened. Measurements of corneal and scleral elasticity are further complicated by the organisation of their fibrillar structure: fibres are oriented in different directions and these directions vary across layers ${ }^{9}$ and with progression from the centre to the periphery. ${ }^{11-12}$

Hjortdal $^{14}$ has addressed some of these points in a very elegant study on 18 human corneae in which he measured the elastic moduli of the different parts of the cornea in both meridional and circumferential directions. He found that in the limbal region, Young's modulus is highest in the circumferential but lowest in the meridional directions and that the highest modulus of all regions and directions was the circumferential limbal value. Differences between circumferential and meridional values were only significant in the paracentral and limbal regions with the latter showing the greatest differences. These results suggest that, with increase in IOP, the limbus is least likely to expand its diameter but may have more flexibility to expand in thickness. It can therefore not only provide support to the corneal curvature, as suggested by Maurice, ${ }^{9}$ it can help to regulate and restrict any shape change that may occur with changes in IOP.

To act as a supporting and adjusting structure for the cornea, Young's modulus of the limbus needs to be carefully balanced with those of the cornea and sclera. If the limbal modulus were much greater than the moduli of the cornea and sclera, the relative rigidity of the limbus could cause disproportionate distensions in the cornea and/or sclera with change in IOP or even with ciliary muscle action during accommodation. Conversely, a relatively low limbal modulus would not offer sufficient support to the cornea, and could result in significant shape change with alterations in IOP. The models tested in this work indicate that the limbal modulus required to maintain a corneal shape for optimal image quality, varies in such a way that there is a peak value which is reached when the ratio of scleral to corneal moduli, $E_{\text {sclera }} / E_{\text {cornear }}$ is around 5-6 for lower corneal eccentricities ( $e=0$ and 0.33 ) and $6-7$ for $e=0.5$ and 0.65 . 
The significance of a peak in limbal modulus at a ratio, $E_{\text {sclera }} / E_{\text {cornea }}$ in the range of $5-7$ is not clear. The only comparative finding in the literature is that of Woo et $a l^{27}$ who reported, from experimental findings, a value of 5 for the ratio of scleral to stromal (corneal) moduli for low levels of effective strain.

Without knowing the values for corneal and scleral moduli, it is not possible to conclude what the optimal modulus value of the limbus may be. However, as the limbus is not composed of tissue that differs from those of the cornea or sclera, its modulus could not be excessively larger or smaller that of the cornea or sclera. If it is to maintain the corneal shape, it should be more rigid that the bulk of the cornea and is more likely to be closer to that of the sclera. This condition is best met for $e=0.5$; at this eccentricity, the peak limbal modulus is closest to that of the sclera for all tested values of the corneal modulus. An eccentricity of 0.5 was also found to be the optimum value for minimising spherical

aberration and enhancing retinal image quality ${ }^{15}$ and it concurs with the findings of Kiely et $a l^{29}$ for mean corneal shape.

From the modelling studies of Liu and Roberts, ${ }^{28}$ within the list of corneal variables for which predicted IOP values coincide with real measurements, a value of $0.19 \mathrm{MPa}$ for the corneal modulus of elasticity is cited. This is close to the value of $0.2 \mathrm{MPa}$ in this study. The peak limbal modulus for a corneal modulus of $0.2 \mathrm{MPa}$ and for $e=0.5$ is around $1.6 \mathrm{MPa}$ (slightly higher for $\mathrm{IOP}=32 \mathrm{mmHg}$ ). The scleral modulus at this point is around $1.2-1.3 \mathrm{Mpa}$, which is close to the reported values of scleral moduli measured from strips of human sclera (1.8 MPa: posterior sclera and $2.9 \mathrm{MPa}$ : anterior sclera) ${ }^{30}$

The models were tested for what is considered a normal, healthy IOP $(16 \mathrm{mmHg})$ as well for low and excessively high IOP values. In the clinical situation, an IOP of $32 \mathrm{mmHg}$ would arouse suspicion of glaucoma but even at a level which is considered pathological, vision may be unaffected suggesting that some selfadjustment of the eyeball may occur. From the models used in this study, the limbal modulus required for preservation of image quality does not vary much with IOP. This indicates that the relationship between stress and strain for the limbal region is linear, over the range of IOP values tested. If corneal curvature is indeed supported by the limbus, this requires the limbus to maintain its elasticity. Nonlinearity in the stress/strain relationship could indicate that the elastic limit of the limbus had been overreached and this could result in corneal deformation with higher IOP.

In some of the figures, the limbal modulus is higher for $\mathrm{IOP}=32 \mathrm{mmHg}$ and this is particularly marked for the model with a spherical cornea $(e=0)$ when $E_{\text {cornea }}=10 \mathrm{MPa}$ (Figure 3c). It is not clear why this high peak value occurs but it does so when the corneal modulus and the IOP are set at extreme values. This may indicate that maintenance of a spherical cornea at such high IOP and corneal moduli values, may be reaching the limits of what is considered physiologically normal.

In the absence of absolute values, the relative moduli of the cornea, sclera, and limbus provide an important guideline for how the material properties of these tissues relate to one another. Young's modulus of the limbal ring needs to be sufficiently strong to maintain corneal shape and yet also flexible enough to permit small adjustments to be made in response to processes that could affect corneal shape and axial length. Further experimental studies are needed to measure the elastic properties of the limbus and to confirm how these may relate to the cornea and sclera. Rheological parameters should be considered in future surgical procedures, particularly those that involve incisions in the limbal area, with the aim of minimising postoperative changes to corneal shape which may adversely affect the refractive status of the eye.

\section{Conclusion}

The eyeball is subjected to small daily variations in IOP and, these processes will exert forces on the outer coat of the eyeball and on the cornea, causing subtle changes in axial length and corneal shape. Such changes must be controlled in order to preserve image quality on the retina. This requires a balance between the rheological properties of the cornea and sclera as well as a stabilising feature that can maintain the corneal shape and help to adjust it in response to IOP changes. Such a role has previously been ascribed to the limbus. This study has found that for maintenance of image quality, there is a peak value for the modulus of elasticity in the limbal region that occurs when the scleral modulus is $5-7$ times that of the corneal. The value of the limbal modulus is closest to that of the sclera for a corneal eccentricity of 0.5. This eccentricity has been found to be the optimum for enhancing retinal image quality.

\section{References}

1 Pierscionek BK, Popiolek-Masajada A, Kasprzak H. Corneal shape change during accommodation. Eye 2001; 15: 766-769.

2 He JC, Gwiazda J, Thorn F, Held R, Huang W. Change in corneal shape and corneal wavefront aberrations with accommodation. J Vis 2003; 3: 456-463.

3 Drexler W, Findl O, Schmetterer L, Hirzenberger CK, Fercher AF. Eye elongation during accommodation in humans: differences between emmetropes and myopes. Invest Ophthalmol Vis Sci 1998; 39: 2140-2147.

4 Walker TW, Mutti DO. The effect of accommodation on ocular shape. Optom Vis Sci 2002; 79: 424-430. 
5 Fledelius HC. Adult onset myopia-oculometric features. Acta Ophthalmol Scand 1995; 73: 397-401.

6 Kinge B, Midelfart A, Jacobsen G, Rystad J. The influence of near-work on development of myopia among university students. A three-year longitudinal study among engineering students in Norway. Acta Ophthalmol Scand 2000; 78: 26-29.

7 Midelfart A, Kinge B, Midelfart S, Lydersen S. Prevalence of refractive errors in young and middle-aged adults in Norway. Acta Ophthalmol Scand 2002; 80: 501-505.

8 Rose K, Smith W, Morgan I, Mitchell P. The increasing prevalence of myopia: implications for Australia. Clin Exp Ophthalmol 2001; 29: 116-120.

9 Maurice DM. Mechanics of the cornea. In: Cavanagh HD (ed). The Cornea. Raven Press: New York, 1988, pp 187-193.

10 Reichel E, Miller D, Blanco E, Mastanduno R. The elastic modulus of the central and perilimbal bovine cornea. Ann Ophthalmol 1989; 21: 205-208.

11 Aghamohammadzadeh H, Newton R, Meek K. X-ray scattering used to map the preferred collagen orientation in the human cornea and limbus. Structure 2004; 12: 249-256.

12 Newton RH, Meek KM. Circumcorneal annulus of collagen fibrils in the human limbus. Invest Ophthalmol Vis Sci 1998; 39: 1125-1134.

13 Dua HS, Azuara-Blanco A. Limbal stem cells of the corneal epithelium. Surv Ophthalmol 2000; 44: 415-425.

14 Hjortdal J. Regional elastic performance of the human cornea. J Biomech 1996; 29: 931-942.

15 Asejczyk-Widlicka M, Srodka W, Kasprzak H, Iskander RD. Influence of IOP on geometrical properties of a linear model of the eyeball. Effect of optical self-adjustment. Optics 2004; 115: 517-524.

16 Kasprzak K, Jankowska-Kuchta E. A new analytical approximation of corneal topography. J. Mod Opt 1996; 43: 1135-1148.

17 MacAdam DL In: Le Grand Y, El Hage SG (eds). Physiological Optics. Springer Verlag: Berlin, 1980.
18 Srodka W, Kasprzak H. Identification of mechanical parameters of incised cornea by means of finite element method. Optica Appl 1996; 26: 9-17.

19 Srodka W, Kasprzak H. The effect of approximating corneal profile on the dynamic changes in refraction. Biol Sport 1997; 14: 324-328.

20 Anderson K, El-Sheikh A, Newson T. Application of structural analysis to the mechanical behaviour of the cornea. J R Soc Lond Interf 2004; 1: 1-13.

21 Andreassen TT, Hjorth Simonsen A, Oxlund H. Biomechanical properties of keratoconus and normal corneas. Exp Eye Res 1980; 31: 435-441.

22 Edmund C. Corneal elasticity and ocular rigidity in normal and keratoconic eyes. Acta Ophthalmol. 1988; 66: 134-140.

23 Hoeltzel DA, Altman P, Buzard K, Choe K. Strip extensiometry for comparison of the mechanical response of bovine, rabbit and human corneas. J Biomech Eng 1992; 114: 202-215.

24 Nyquist GW. Rheology of the cornea: experimental techniques and results. Exp Eye Res 1968; 7: 183-188.

25 Orssengo GJ, Pye DC. Determination of the true intraocular pressure and modulus of elasticity of the human cornea in vivo. Bull Math Biol 1999; 61: 551-572.

26 Sjøntoft E, Edmund C. In vivo determination of Young's modulus for the human cornea. Bull Math Biol 1987; 49: 217-232.

27 Woo SL, Kobayashi AS, Lawrence C, Schlegel WA. Nonlinear material properties of intact cornea and sclera. Exp Eye Res 1972; 14: 29-39.

28 Liu JL, Roberts CJ. Influence of corneal biomechanical properties on intraocular pressure measurement. Quantitative analysis. J Cataract Refract Surg 2005; 31: 146-155.

29 Kiely MP, Smith GM, Carney L. The mean shape of the human cornea. Optica Acta 1982; 29: 1027-1040.

30 Friberg TR, Lace JW. A comparison of the elastic properties of human choroid and sclera. Exp Eye Res 1988; 47: 429-436. 